Dudek Iga, Sokół Dorota, Skubel Tomasz, Dobrzyński Michał, Drozd Malgorzata, Czarnota Jakub, Forma Alicja, Piecewicz-Szczęsna Halina. The risk of developing psoriasis in alcohol-dependent patients and possible therapeutic strategies. The literature review. Journal of Education, Health and Sport. 2020;10(8):450-457. eISSN 2391-8306. DOI http://dx.doi.org/10.12775/JEHS.2020.10.08.055

https://apcz.umk.pl/czasopisma/index.php/JEHS/article/view/JEHS.2020.10.08.055

https://zenodo.org/record/4009413

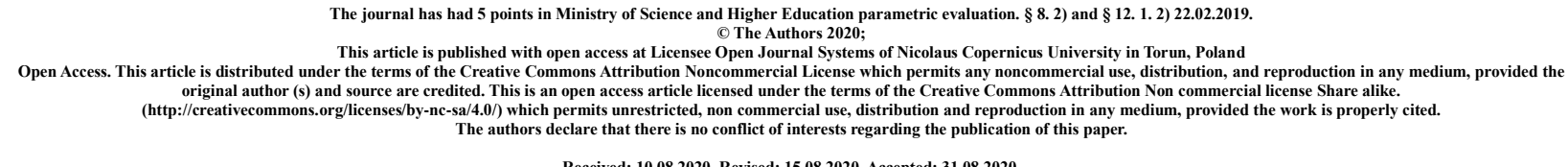

Recrived: 10.082020 . Revised: 15.08 .2020 . Acepted: 31.082020.

\title{
The risk of developing psoriasis in alcohol-dependent patients and possible therapeutic strategies
}

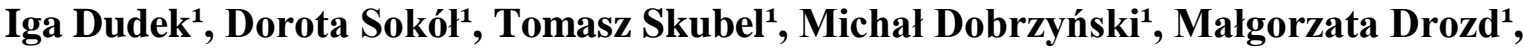 \\ Jakub Czarnota1, Alicja Forma1, Halina Piecewicz-Szczęsna²
}

${ }^{1}$ Student Research Group at the Department of Epidemiology and Clinical Research

Methodology, Medical University of Lublin

${ }^{2}$ Department of Epidemiology and Clinical Research Methodology, Medical University of Lublin Supervisor: dr n. med. Halina Piecewicz-Szczęsna

Corresponding author: Halina Piecewicz-Szczęsna, e-mail: halpiec@wp.pl

\section{ORCID ID:}

Iga Dudek: orcid.org/0000-0002-8101-074X; iga.dudek6@gmail.com

Dorota Sokół: orcid.org/0000-0003-2059-7951; dorota.soko19606@gmail.com

Tomasz Skubel: orcid.org/0000-0001-7572-401X; tomasz.wojciech.skubel@gmail.com

Michał Dobrzyński: orcid.org/0000-0002-1416-6568; mdobrzyski4@ gmail.com

Małgorzata Drozd: orcid.org/0000-0002-0710-2451; drozd.malg@ gmail.com

Jakub Czarnota: orcid.org/0000-0003-2783-0349; kubbaa232@ interia.pl

Alicja Forma: orcid.org/0000-0001-8714-7627; aforma@ onet.pl

Halina Piecewicz-Szczęsna: orcid.org/0000-0002-0573-7226 


\begin{abstract}
:
Excessive alcohol consumption significantly contributes to the damage of the nervous, cardiovascular and digestive systems. According to the latest scientific reports, excessive ethanol consumption might also contribute to an increased risk of developing skin diseases such as rosacea, porphyria cutanea tarda or psoriasis. The risk of developing psoriasis in alcoholdependent patients is estimated at 3.1-3.4\%. Among these people, in addition to the use of traditional therapeutic strategies, therapy with drugs acting on the neurotransmitter systems may constitute a potential treatment. Chronic alcohol consumption increases the activation of $\mathrm{T}$ lymphocytes as well as the level of tumor necrosis factor- $\alpha$ (TNF- $\alpha$ ) and also stimulates the excessive proliferation of keratinocytes. These factors dysregulate the immune system, predisposing to the risk of developing psoriasis. Alcohol also interferes with signaling through neurotransmission affecting dopaminergic and serotonergic systems, which may affect the immune processes in the pathogenesis of psoriasis. The results of the research showed that the daily consumption of alcohol in an amount greater than 80 grams significantly reduces the effectiveness of treatment in psoriatic patients. Moreover, traditional drugs used in the course of psoriasis (retinoids, methotrexate, cyclosporine) may show reduced activity or even lead to toxic side effects (liver fibrosis) if alcohol is not discontinued during therapy. Treatment of psoriatic patients and coexisting alcohol dependence is a separate therapeutic problem. In addition to traditional methods, drugs that affect the neurotransmitter system may become a chance, mainly due to the influence of alcohol on these systems and modulation of the immune response, which is important in the development of psoriasis.
\end{abstract}

Key words: psoriasis; alcohol; psoriasis treatment

\title{
Introduction
}

Psoriasis is a chronic, autoimmune disease of the skin, nails, and joints that affects over 125 million people worldwide. [1] It can develop at any age, but it is most common between 18 and 26 years of age and between 50 and 69 years of age. [1] [2] Although it occurs with the same frequency in both sexes, it is believed that males might be exposed to a more aggressive course of the disease. [3] In terms of risk factors, it is estimated that $50-70 \%$ of the susceptibility to developing the disease is due to a genetic variation of more than 40 genome sites and more than 400 single nucleotide polymorphisms (SNPs). [4] These variations are mainly observed in genes encoding interleukin 2 (IL-2), major histocompatibility complex (MHC), interleukin 23 receptor (IL-23R), interleukin 21 (IL-21) and IL-23R antagonist. In people with genetic susceptibility, environmental factors such as infections, certain medications, such as lithium, smoking, and stress can be a trigger factor in the development of the disease. [5] One of the modifiable risk factors is excessive alcohol consumption. [6] It affects both the manifestation of the disease and its more severe course. The pathogenesis of psoriasis is not completely understood. Currently, it is assumed that the over-activation of the immune system plays a major role. [5] 
Activated macrophages, natural T killer cells, or plasmacytoid dendritic cells indirectly stimulate myeloid dendritic cells, which secrete cytokines leading to excessive keratinocyte proliferation and infiltration of inflammatory cells into the skin. Five types of psoriasis have been described: psoriasis vulgaris, guttate psoriasis, inverse psoriasis, pustular psoriasis and erythrodermic psoriasis. [3] Erythrodermic psoriasis is a severe and rare form of psoriasis affecting $90 \%$ of the body surface and can be life-threatening. [1] There is a risk of developing hypothermia, hypoalbuminemia, and heart failure. [7] The most common type of psoriasis is psoriasis vulgaris, which accounts for $90 \%$ of cases of this disease. There are red or salmon pink plaques which are covered by silvery scales that are symmetrically arranged and appear especially on the scalp, extensor aspects of knees and elbows, umbilicus, and lumbosacral region. As for psoriasis arthritis, it occurs in 6-42\% of psoriasis patients. [8] It is arthritis classified as spondyloarthritis by correlation with HLA-B27, presence of spondylitis and extra-articular features like iriditis, urethritis, diarrhea and aortic root dilatation.

\section{Ethanol and its effects on skin}

Ethanol is a substance that is widely consumed in nearly all of the regions in the world. It affects many organ systems and contributes to carcinogenesis, the development of liver cirrhosis, arterial hypertension, ischemic and hemorrhagic stroke, and also to coronary heart disease. [9] The toxic effects of alcohol consumption are mainly due to metabolism to acetaldehyde, NAD + depletion and formation of reactive nitrogen, and oxygen species. [10] Acetaldehyde and oxidative induce the damage of the DNA, proteins, and lipids. Due to alcohol violation by gastrointestinal mucosal barrier and translocation of endotoxins and other molecules via portal blood pathway to the liver, the innate immune response is activated. The adaptive immune response is stimulated by acetaldehyde-protein products. Ethanol can also directly alter the functions of $\mathrm{T}$ lymphocytes and natural killer cells. [11] Taken together, this leads to a decrease in immunity and an abnormal immune response to pathogens, resulting in more frequent infections and longer recovery. After ingestion, ethanol is subject to the first-pass metabolism in the stomach and enters the liver with portal blood. [12] In the liver, alcohol is metabolized to acetaldehyde by dehydrogenase. The next step is the oxidation of acetaldehyde to acetate, which in turn can be converted into $\mathrm{CO} 2$, fatty acids, cholesterol, ketone bodies, or steroids. Ingested ethanol can be removed through the skin by active transport, mainly through the sweat glands, and by passive diffusion through the skin's lipid membranes. [13] The secretion of alcohol through the lipid barrier causes epidermal barrier damage which increases the permeability of numerous chemicals. The entry of exogenous factors can lead to the activation of keratinocytes and the secretion of cytokines leading to the development of inflammation. These elements are crucial in the pathogenesis of psoriasis. Ethanol and acetone directly stimulate the proliferation of keratinocytes and also increase the amount of mRNA of the genes such as $\alpha-5$ integrin, cyclin D1, and keratinocyte growth factor receptor (KGFR), which stimulate proliferation. [14] High levels of ethanol cause upregulation of TACE (tumor necrosis factor- $\alpha$ converting enzyme) in lymphocytes and peripheral blood monocytes, increasing TNF- $\alpha$. 
This factor also contributes to the proliferation of keratinocytes. [13] Reactive oxygen species also contribute to psoriasis by modulating signal transduction pathways and overexpressing chemokines such as interleukin 6 (IL-6), interleukin 1 (IL-1), TNF- $\alpha$ and interleukin 8 (IL-8).

\section{Alcohol as a risk factor}

The role of alcohol as a risk factor for psoriasis has been debated in many studies. Most of them confirm the link between alcohol abuse and the development of the disease. [15] [16] [17] A meta-analysis of 15 case-control studies showed a strong correlation between the risk of psoriasis and alcohol consumption ( $\mathrm{OR}=1.531,95 \% \mathrm{CI}=1.164-2.014, \mathrm{p}=0.002)$. [18] A study of 82,869 women in the US found that the appearance of psoriasis depended on the amount of alcohol consumed and the type of alcoholic beverage. [19] The risk of developing the disease was 2.3 times greater in women who consumed 5 or more nonlight beers per week. In a study by Gupta et al, alcohol consumption of more than $80 \mathrm{~g} /$ day among men with psoriasis was correlated with worse response to treatment. [20] Patients with moderate to severe disease have a higher risk of death, and alcohol is a major contributor to the increase in mortality. [21] Moreover, it has been shown that psoriasis patients have a $60 \%$ higher risk of dying from alcohol-related causes. [22] The distribution of psoriasis plaques in people consuming ethanol concerns mainly acral surfaces and the plaques are hyperkeratotic, flatter and erythematous. [23] This distribution is characteristic of HIV patients, suggesting a role for alcohol in inducing immunosuppression. [24] Patients with psoriasis are also more likely to develop alcohol dependence and more often than the healthy population have a type D personality ("distressed"), which is characterized by a tendency to inhibit behavior and emotions in the community and by frequent negative emotions. [25]

\section{Treatment possibilities}

The choice of therapy against psoriasis depends primarily on its severity. For mild psoriasis, that typically involves less than 3-5\% body surface area (BSA), the treatment options are topical medications such as steroid ointments, vitamin D analogues (calcitriol, calcipotriol, calcipotriene), calcineurin inhibitors (tacrolimus, pimecrolimus), keratinolytics (tazarotene, salicylic acid) and targeted phototherapy. [5] Systemic therapy is intended for the treatment of moderate to severe psoriasis. It includes the use of drugs from four groups of biological drugs, which are TNF- $\alpha$, IL-23, IL-17 or IL-12/23 inhibitors, oral agents such as cyclosporine, methotrexate, apremilast and acitretin or the use of PUVA therapy using UVA light and psoralen. Excessive alcohol consumption complicates the choice of appropriate treatment in terms of toxicity, drug interactions and the worsening of psoriasis. For example, ethanol converts acitretin to etretinate, a teratogenic substance that can accumulate in the body's fat tissue up to three years after stopping treatment. [26] For this reason, alcohol consumption with acitretin therapy is contraindicated, and women should use effective contraception while taking the drug and for 3 years after its discontinuation. It has also been shown that acute ethanol exposure may contribute to the inhibition of the enzyme CYP3A4, which is involved in the metabolism of cyclosporine, resulting in its accumulation. [27] 
Methotrexate (MTX), a folic acid antagonist, reduces DNA and RNA synthesis in activated T cells and keratinocytes, resulting in the relief of psoriasis symptoms. [28] The main side effect of methotrexate is its hepatotoxicity. If treatment is long, ultrasound and liver biopsy are required to assess the extent of organ damage. [29] Psoriatic patients treated with methotrexate are at risk of liver damage twice as often as patients treated for rheumatoid arthritis. [30] It is therefore believed that these individuals may be particularly sensitive to the toxic effects of MTX. The explanation may be the fact that in the course of psoriasis, regardless of treatment, up to half of the patients develop nonalcoholic fatty liver disease. [31] [32] In a study of patients with chronic plaque psoriasis, the incidence of NAFLD in these patients was $43 \%$ compared to the control group (28\%). [33] None of these patients were treated with a drug causing liver toxicity, and the development of NAFLD was correlated with the severity of psoriasis. Ethanol consumption is contraindicated during methotrexate therapy due to the hepatotoxicity of both substances. [29] For example, a 2007 study found that all psoriasis patients treated with MTX and consumed high doses of alcohol ( $>30 \mathrm{~g} /$ day) developed liver fibrosis. [34] Also, therapy based on biological treatment with TNF- $\alpha$ inhibitors, especially with infliximab, is associated with hepatotoxicity. [35] It has sometimes been associated with concomitant MTX treatment or reactivation of viral hepatitis, especially hepatitis B. There have been reports of jaundice and hepatitis not related to infection, and even liver failure requiring transplantation with infliximab therapy. [36] Etanercept is an example of a TNF- $\alpha$ inhibitor that does not damage the liver [35] but in clinical trials, it was the least effective of this group of drugs in reducing psoriasis. Interestingly, despite the hepatotoxicity of drugs from the group of TNF- $\alpha$ inhibitors, they may exert a positive role in alcoholic hepatitis, but due to the lack of high-quality research on this topic, it is not possible to draw firm conclusions. [35] Separate treatment algorithms have been developed for the treatment of patients with psoriasis associated with alcoholism. [37] In the treatment of mild disease, steroid ointments, vitamin D analogues, tacrolimus, and tazatorene should be used. First-line therapy in the extensive forms is PUVA, narrow-band ultraviolet B (NB-UVB), and treatment with adalimumab and infliximab (TNF- $\alpha$ inhibitors). Acitretin is one of the second-line treatment options, while methotrexate is intended for the treatment of refractory cases as the last line treatment.

\section{Neurotransmitter agents}

According to the latest research, in the course of psoriasis, receptors for biogenic amines, such as acetylcholine, 5-HT and tachykinin receptors in the basal epidermal cells are overexpressed. [38] [39] [40] Therefore, drugs that act on amines may modulate the formation of psoriatic eruptions. One cohort study showed that patients who received treatment with SSRIs (serotonin reuptake inhibitors) were less likely to require systemic therapy for psoriasis. [41] A reduction in the area of the skin affected by psoriasis has been observed in people taking the antidepressant drug bupropion, which is a norepinephrine-dopamine reuptake inhibitor. Levodopa, a dopamine precursor used in the treatment of Parkinson's disease, also contributed to the relief of psoriasis symptoms. These drugs are also used in the treatment of alcohol dependence, the basis of which is the development of neurochemical changes in the brain. [42] 
These changes include a reduction in the level of neurotransmitters such as GABA, serotonin or dopamine, and an increase in glutamate, norepinephrine or the activity of the hypothalamicpituitary axis.

\section{Conclusions}

Alcohol is a risk factor for the development of psoriasis and may worsen the disease by modulating the immune response that appears to be of key importance in the pathogenesis of psoriasis. More research is needed to confirm the negative effects of alcohol on this disease. Modification of treatment in patients with psoriasis and alcohol dependence appears to play a key role due to the possible negative drug-alcohol interactions and the potential for dangerous side effects. Every person with psoriasis requires a careful medical history and consideration of possible excessive alcohol consumption, which is more common in this group of patients than in the rest of the population. Drugs that act on neurotransmitters may improve the effects of treatment since a reduction in psoriatic lesions has been observed in studies using them. These drugs also act on the neurogenic basis of alcohol addiction, which may bring additional benefit and synergism in the treatment of both of these diseases.

\section{List of references}

[1] Greb, J. E., Goldminz, A. M., Elder, J. T., Lebwohl, M. G., Gladman, D. D., Wu, J. J., ... Gottlieb, A. B. (2016). Psoriasis. Nature Reviews Disease Primers, 2, 16082.

[2] Parisi, R. et al. Global epidemiology of psoriasis: a systematic review of incidence and prevalence. J. Invest. Dermatol. 133, 377-385 (2013).

[3] Boehncke, W.-H., \& Schön, M. P. (2015). Psoriasis. The Lancet, 386(9997), 983-994.

[4] Nussbaum, L., Chen, Y. L., \& Ogg, G. S. (2020). Role of regulatory T cells in psoriasis pathogenesis and treatment. British Journal of Dermatology.

[5] Armstrong AW, Read C. Pathophysiology, Clinical Presentation, and Treatment of Psoriasis: A Review. JAMA. 2020;323(19):1945-1960.

[6] Kovitwanichkanont T, Chong AH, Foley P. Beyond skin deep: addressing comorbidities in psoriasis. Med J Aust. 2020;212(11):528-534.

[7] Griffiths, C. E., \& Barker, J. N. (2007). Pathogenesis and clinical features of psoriasis. The Lancet, 370(9583), 263-271.

[8] Gladman, D. D. (2005). Psoriatic arthritis: epidemiology, clinical features, course, and outcome. Annals of the Rheumatic Diseases, 64(suppl_2), ii14-ii17.

[9] Corrao, G. (2004). A meta-analysis of alcohol consumption and the risk of 15 diseases. Preventive Medicine, 38(5), 613-619.

[10] Rusyn, I., \& Bataller, R. (2013). Alcohol and toxicity. Journal of Hepatology, 59(2), 387388.

[11] Szabo G, Mandrekar P. A recent perspective on alcohol, immunity, and host defense. Alcohol Clin Exp Res. 2009;33(2):220-232.

[12] Cederbaum, A. I. (2012). Alcohol Metabolism. Clinics in Liver Disease, 16(4), 667-685. 
[13] Farkas, Á., \& Kemény, L. (2013). Alcohol, Liver, Systemic Inflammation and Skin: A Focus on Patients with Psoriasis. Skin Pharmacology and Physiology, 26(3), 119-126.

[14] Farkas A, Kemeny L, Szell M, Dobozy A, BataCsorgo Z: Ethanol and acetone stimulate the proliferation of $\mathrm{HaCaT}$ keratinocytes: the possible role of alcohol in exacerbating psoriasis. Arch Dermatol Res 2003;295:56-62.

[15] Higgins EM, du Vivier AW. Alcohol and the skin. Alcohol Alcohol 27(6):595-602 (1992 Nov).

[16] Higgins EM, du Vivier AW. Cutaneous disease and alcohol misuse. Br Med Bull 50(1):8598 (1994 Jan).

[17] Poikolainen K, Reunala T, Karvonen J, et al. Alcohol intake: a risk factor for psoriasis in young and middle aged men? BMJ 300(6727):780-3 (1990 Mar 24).

[18] ZHU, K.-J., ZHU, C.-Y., \& FAN, Y.-M. (2012). Alcohol consumption and psoriatic risk: A meta-analysis of case-control studies. The Journal of Dermatology, 39(9), 770-773.

[19] Qureshi, A. A., Dominguez, P. L., Choi, H. K., Han, J., \& Curhan, G. (2010). Alcohol Intake and Risk of Incident Psoriasis in US Women. Archives of Dermatology, 146(12), 1364. [20] Gupta, M. A., Schork, N. J., Gupta, A. K., \& Ellis, C. N. (1993). Alcohol intake and treatment responsiveness of psoriasis: A prospective study. Journal of the American Academy of Dermatology, 28(5), 730-732.

[21] Poikolainen K, Karvonen J, Pukkala E. Excess mortality related to alcohol and smoking among hospital-treated patients with psoriasis. Arch Dermatol. 1999;135(12):1490-1493. [22] Parisi R, Webb RT, Carr MJ, Moriarty KJ, Kleyn CE, Griffiths CEM, et al. Alcohol-related mortality in patients with psoriasis: a population-based cohort study. JAMA Dermatol 2017; 153:1256-62.

[23] Farber E, Nall L. Psoriasis and alcoholism. Cutis 1994;53:21-7.

[24] Cockerell CJ. Cutaneous manifestations of HIV infection other than Kaposi's sarcoma: clinical and histological aspects. J Am Acad Dermatol 1990;22:1260-9.

[25] Lim DS, Bewley A, Oon HH. Psychological Profile of Patients with Psoriasis. Ann Acad Med Singapore. 2018;47(12):516-522.

[26] Ormerod, A. D., Campalani, E., \& Goodfield, M. J. D. (2010). British Association of Dermatologists guidelines on the efficacy and use of acitretin in dermatology. British Journal of Dermatology, 162(5), 952-963.

[27] Vena, G. A., \& Cassano, N. (2012). The effects of alcohol on the metabolism and toxicology of anti-psoriasis drugs. Expert Opinion on Drug Metabolism \& Toxicology, 8(8), 959-972.

[28] Balak, D. M. W., Gerdes, S., Parodi, A., \& Salgado-Boquete, L. (2020). Long-term Safety of Oral Systemic Therapies for Psoriasis: A Comprehensive Review of the Literature. Dermatology and Therapy.

[29] Hannoodee M, Mittal M. Methotrexate. [Updated 2020 Apr 7]. In: StatPearls [Internet]. Treasure Island (FL): StatPearls Publishing; 2020 Jan.

[30] Kim, N., Thrash, B., \& Menter, A. (2010). Comorbidities in Psoriasis Patients. Seminars in Cutaneous Medicine and Surgery, 29(1), 10-15. 
[31] Gisondi P, Del Giglio M, Cozzi A, Girolomoni G. Psoriasis, the liver, and the gastrointestinal tract. Dermatol Ther. 2010;23(2):155-159.

[32] Mantovani A, Gisondi P, Lonardo A, Targher G. Relationship between Non-Alcoholic Fatty Liver Disease and Psoriasis: A Novel Hepato-Dermal Axis?. Int J Mol Sci. 2016;17(2):217.

Published 2016 Feb 5.

[33] Gisondi, P., Targher, G., Zoppini, G., \& Girolomoni, G. (2009). Non-alcoholic fatty liver disease in patients with chronic plaque psoriasis. Journal of Hepatology, 51(4), 758-764.

[34] Rosenberg P, Urwitz H, Johannesson A, et al. Psoriasis patients with diabetes type 2 are at high risk of developing liver fibrosis during methotrexate treatment. J Hepatol. 2007;46(6):11111118.

[35] Cassano, N., Vestita, M., Apruzzi, D., \& Vena, G. A. (2011). Alcohol, psoriasis, liver disease, and anti-psoriasis drugs. International Journal of Dermatology, 50(11), 1323-1331. [36] European Medicines Agency. Infliximab Product Information. European Medicines Agency, 2010. http://www.ema.europa.eu/ (accessed June 30, 2011).

[37] Kim, H. J., \& Lebwohl, M. G. (2018). Biologics and Psoriasis. Dermatologic Clinics. [38] Nordlind K, Azmitia EC, Slominski A. The skin as a mirror of the soul: exploring the possible roles of serotonin. Exp Dermatol. 2008;17(4):301-311.

[39] Remröd C, Lonne-Rahm S, Nordlind K. Study of substance P and its receptor neurokinin-1 in psoriasis and their relation to chronic stress and pruritus. Arch Dermatol Res. 2007;299(2):8591.

[40] Radosa J, Dyck W, Goerdt S, Kurzen H. The cholinergic system in guttate psoriasis with special reference to mast cells. Exp Dermatol. 2011;20(8):677-679.

[41] Svanström C, Lonne-Rahm SB, Nordlind K. Psoriasis and alcohol. Psoriasis (Auckl). 2019;9:75-79.

[42] Becker, H. C., \& Mulholland, P. J. (2014). Neurochemical mechanisms of alcohol withdrawal. Alcohol and the Nervous System, 133-156. 